\title{
Betti numbers of strongly color-stable ideals and squarefree strongly color-stable ideals
}

\author{
Satoshi Murai
}

Received: 6 March 2007 / Accepted: 7 August 2007 / Published online: 13 September 2007

(C) Springer Science+Business Media, LLC 2007

\begin{abstract}
In this paper, we will show that the color-squarefree operation does not change the graded Betti numbers of strongly color-stable ideals. In addition, we will give an example of a nonpure balanced complex which shows that colored algebraic shifting, which was introduced by Babson and Novik, does not always preserve the dimension of reduced homology groups of balanced simplicial complexes.
\end{abstract}

Keywords Colored algebraic shifting · Balanced complexes · Graded Betti numbers

\section{Introduction}

In the present paper, we study the graded Betti numbers of strongly color-stable ideals and squarefree strongly color-stable ideals introduced in [5], and show that the graded Betti numbers of a strongly color-stable ideal are equal to those of some squarefree strongly color-stable ideal.

Algebraic shifting, which was introduced by Kalai, is a map that associates with each simplicial complex another simplicial complex having a simpler structure, called shifted. Algebraic shifting was used to give several remarkable results in the theory of face numbers of simplicial complexes, such as the characterization of pairs of face numbers and Betti numbers (i.e., the dimension of reduced homology groups) of simplicial complexes (Björner and Kalai [8]). On the other hand, balanced complexes were introduced by Stanley [17], and face vectors of balanced complexes have been well studied. (See e.g., [7, 12, 17] and [18].) Since algebraic shifting is not effective for balanced complexes because most of the shifted complexes are not balanced, it

The author is supported by JSPS Research Fellowships for Young Scientists.

\footnotetext{
S. Murai $(\bowtie)$

Department of Pure and Applied Mathematics, Graduate School of Information Science

and Technology, Osaka University, Toyonaka, Osaka 560-0043, Japan

e-mail: s-murai@ist.osaka-u.ac.jp
} 
was asked in [15, Problem 48] to extend algebraic shifting to balanced complexes and characterize pairs of face numbers and Betti numbers of balanced complexes.

For this problem, Babson and Novik [5] introduced a new operation, called colored algebraic shifting, which associates with each balanced complex another balanced complex having a simpler structure, called color-shifted. We will study color-shifted complexes and the color-squarefree operation which plays an important role in the theory of colored algebraic shifting.

Let $K$ be an infinite field and $V$ a set of variables. Write $K[V]$ for the polynomial ring over the field $K$ in the set of variables $V$ and $\mathcal{M}[V]$ for the set of monomials in $K[V]$. For each monomial $x_{1}^{a_{1}} \cdots x_{k}^{a_{k}} \in \mathcal{M}[V]$ where each $x_{j} \in V$, the integer $\operatorname{deg}\left(x_{1}^{a_{1}} \cdots x_{k}^{a_{k}}\right)=\sum_{j=1}^{k} a_{j}$ will be called the standard degree of $x_{1}^{a_{1}} \cdots x_{k}^{a_{k}}$. Assume that $V$ is a finite set endowed with an ordered partition $\left(V_{1}, V_{2}, \ldots, V_{r}\right)$, that is, $V$ is a set of the form $V=\dot{U}_{j=1}^{r} V_{j}$, where $\dot{U}$ denotes a disjoint union. Set $\left|V_{j}\right|=n_{j}$ and $V_{j}=\left\{x_{j, 1}, x_{j, 2}, \ldots, x_{j, n_{j}}\right\}$ for all $j$, where $|A|$ denotes the cardinality of a finite set $A$. For any monomial $u=\Pi_{j=1}^{r}\left(x_{j, 1}^{a_{j, 1}} \cdots x_{j, n_{j}}^{a_{j, n_{j}}}\right) \in \mathcal{M}[V]$, we write

$$
\operatorname{Deg}_{j}(u)=a_{j, 1}+a_{j, 2}+\cdots+a_{j, n_{j}}
$$

and

$$
\operatorname{Deg}(u)=\left(\operatorname{Deg}_{1}(u), \operatorname{Deg}_{2}(u), \ldots, \operatorname{Deg}_{r}(u)\right) \in \mathbb{Z}^{r} .
$$

The above $\operatorname{Deg}(u) \in \mathbb{Z}^{r}$ will be called the multidegree of $u$. Define the $\mathbb{Z}^{r}$-grading of $K[V]$ by using multidegree, and define the $\mathbb{Z}$-grading of $K[V]$ by using the standard degree. We simply say 'graded' if we consider the $\mathbb{Z}$-grading.

A multicomplex $M$ on $V$ is a set of monomials in $K[V]$ such that if $u \in M$ and $v$ divides $u$ then $v \in M$. A multicomplex $M$ is called a simplicial complex if all monomials in $M$ are squarefree.

Let $\Gamma$ be a simplicial complex on $V$. The elements of $\Gamma$ are called faces, and the maximal ones (under divisibility) are called facets. The dimension of $\Gamma$ is the integer $\operatorname{dim} \Gamma=\max \{\operatorname{deg}(u): u \in \Gamma\}-1$. Let $f_{i}(\Gamma)$ be the number of monomials $u \in \Gamma$ of degree $i+1$. The vector $f(\Gamma)=\left(f_{-1}(\Gamma), f_{0}(\Gamma), \ldots, f_{\operatorname{dim} \Gamma}(\Gamma)\right)$ will be called the $f$-vector of $\Gamma$. Also, for $\mathbf{c}=\left(c_{1}, \ldots, c_{r}\right) \in \mathbb{N}^{r}$, let $f_{\mathbf{c}}(\Gamma)$ be the number of monomials $u \in \Gamma$ with $\operatorname{Deg}(u)=\mathbf{c}$. The vector $\left(f_{\mathbf{c}}(\Gamma): \mathbf{c} \in \mathbb{N}^{r}\right)$ is called the flag $f$-vector of $\Gamma$. Let $\mathbf{a}=\left(a_{1}, \ldots, a_{r}\right) \in \mathbb{Z}_{>0}^{r}$. A simplicial complex $\Gamma$ on $V$ is said to be a-balanced if $\operatorname{dim} \Gamma+1=\sum_{j=1}^{r} a_{j}$ and $\operatorname{Deg}_{j}(u) \leq a_{j}$ for all $j=1,2, \ldots, r$ and for all $u \in \Gamma$. In particular $\Gamma$ is said to be completely balanced if $\mathbf{a}=(1,1, \ldots, 1)$.

We define the partial order $<_{\mathrm{P}}$ on $\mathcal{M}\left[V_{j}\right]$ by

$$
x_{j, s_{1}} x_{j, s_{2}} \cdots x_{j, s_{k}} \leq P x_{j, t_{1}} x_{j, t_{2}} \cdots x_{j, t_{l}} \Leftrightarrow k \leq l \quad \text { and } \quad s_{i} \leq t_{i} \text { for } i=1,2, \ldots, k
$$

where $s_{1} \leq \cdots \leq s_{k}$ and $t_{1} \leq \cdots \leq t_{l}$. Extend the partial order $<_{\mathrm{P}}$ to $\mathcal{M}[V]$ by setting $u_{1} u_{2} \cdots u_{r} \leq_{\mathrm{P}} v_{1} v_{2} \cdots v_{r}$ if $u_{j} \leq_{\mathrm{P}} v_{j}$ for all $j$, where $u_{j}$ and $v_{j}$ are monomials in $\mathcal{M}\left[V_{j}\right]$. A monomial ideal $I \subset K[V]$ is said to be strongly color-stable if, for all monomials $u \in I$ and $v \leq_{\mathrm{P}} u$ with $\operatorname{Deg}(v)=\operatorname{Deg}(u)$, it follows that $v \in I$. Set

$$
\mathcal{A}=\left\{u_{1} u_{2} \cdots u_{r}: u_{j} \in \mathcal{M}\left[V_{j}\right] \quad \text { and } \quad \operatorname{deg}\left(u_{j}\right)+\max \left(u_{j}\right) \leq n_{j}+1 \text { for all } j\right\}
$$


where $\max \left(u_{j}\right)=\max \left\{t: x_{j, t}\right.$ divides $\left.u\right\}$. The color-squarefree operation $\tilde{\Phi}: \mathcal{A} \rightarrow$ $\mathcal{M}[V]$ is the map defined by

$$
\begin{aligned}
& \tilde{\Phi}\left(\prod_{j=1}^{r}\left(x_{j, s_{(j, 1)}} x_{j, s_{(j, 2)}} \cdots x_{j, s_{\left(j, k_{j}\right)}}\right)\right) \\
& \quad=\prod_{j=1}^{r}\left(x_{j, s_{(j, 1)}} x_{j, s_{(j, 2)}+1} x_{j, s_{(j, 3)}+2} \cdots x_{j, s_{\left(j, k_{j}\right)}+k_{j}-1}\right)
\end{aligned}
$$

where $s_{(j, 1)} \leq \cdots \leq s_{\left(j, k_{j}\right)}$ for all $j$. Note that $\tilde{\Phi}$ gives a one-to-one correspondence between $\mathcal{A}$ and the set of squarefree monomials in $K[V]$. Let $I \subset K[V]$ be a monomial ideal. Then there exists the minimal set of monomials which generates $I$. We write $\operatorname{Gen}(I)$ for the set of minimal monomial generators of $I$. If $\operatorname{Gen}(I) \subset \mathcal{A}$, then write $\tilde{\Phi}(I)$ for the squarefree monomial ideal generated by $\{\tilde{\Phi}(u): u \in \operatorname{Gen}(I)\}$.

The Stanley-Reisner ideal $I_{\Gamma} \subset K[V]$ of a simplicial complex $\Gamma$ on $V$ is the monomial ideal generated by all squarefree monomials $u \notin \Gamma$. Let $G=G L_{n_{1}}(K) \times$ $G L_{n_{2}}(K) \times \cdots \times G L_{n_{r}}(K)$ where each $G L_{n_{j}}(K)$ is the general linear group with coefficients in $K$. Roughly speaking, colored algebraic shifting is defined as follows: assume that $\operatorname{char}(K)=0$ from now on. Fix a total order $\prec$ on $V$ satisfying $x_{j, 1} \succ x_{j, 2} \succ \cdots \succ x_{j, n_{j}}$ for all $j$. The reverse lexicographic order $\prec_{\text {rev }}$ induced by $\prec$ is the total order on $\mathcal{M}[V]$ defined as follows, for all monomials $u=x_{i_{1}, j_{1}} \cdots x_{i_{k}, j_{k}} \in \mathcal{M}[V]$ and $v=x_{i_{1}^{\prime}, j_{1}^{\prime}} \cdots x_{i_{\ell}^{\prime}, j_{\ell}^{\prime}} \in \mathcal{M}[V]$ with $x_{i_{1}, j_{1}} \succeq \cdots \succeq x_{i_{k}, j_{k}}$ and $x_{i_{1}^{\prime}, j_{1}^{\prime}} \succeq \cdots \succeq x_{i_{\ell}^{\prime}, j_{\ell}^{\prime}}$, one has $u \succ_{\text {rev }} v$ if $k>\ell$ or $k=\ell$ and there exists $1 \leq r \leq k$ such that $x_{i_{r}, j_{r}} \succ x_{i_{r}^{\prime}}, j_{r}^{\prime}$ and $x_{i_{t}, j_{t}}=x_{i_{t}^{\prime}}, j_{t}^{\prime}$ for all $t>r$. Choose a generic matrix $\varphi$ of $G$ and consider the initial ideal in ${ }_{\prec} \varphi\left(I_{\Gamma}\right)$ with respect to the reverse lexicographic order induced by $\prec$. This initial ideal is called the $G$-generic initial ideal of I with respect to $\prec$. $G$-generic initial ideals are strongly color-stable, and satisfy $\operatorname{Gen}\left(\operatorname{in}_{\prec} \varphi\left(I_{\Gamma}\right)\right) \subset \mathcal{A}$. Colored algebraic shifting (with respect to $\prec$ ) is the map $\Gamma \rightarrow \tilde{\Delta}_{\prec}(\Gamma)$ defined by $I_{\tilde{\Delta}_{\prec}(\Gamma)}=\tilde{\Phi}\left(\operatorname{in}_{\prec} \varphi\left(I_{\Gamma}\right)\right)$. (The precise definition of $\tilde{\Delta}_{\prec}(\Gamma)$ will be given in Sect. 1.)

Let $\Gamma$ be a simplicial complex on $V$. The following properties appeared in [5]:

(C1) $\tilde{\Delta}_{\prec}(\Gamma)$ is color-shifted, that is, if $u \in \tilde{\Delta}_{\prec}(\Gamma)$ and $v \in \mathcal{M}[V]$ are squarefree monomials satisfying $v \geq_{\mathrm{P}} u$ and $\operatorname{Deg}(v)=\operatorname{Deg}(u)$ then $v \in \tilde{\Delta}_{\prec}(\Gamma)$;

(C2) $\Gamma$ and $\tilde{\Delta}_{\prec}(\Gamma)$ have the same flag $f$-vector;

(C3) If $\Gamma \subset \Sigma$ then $\tilde{\Delta}_{\prec}(\Gamma) \subset \tilde{\Delta}_{\prec}(\Sigma)$.

Colored algebraic shifting behaves nicely for balanced complexes. For example, (C2) says that if $\Gamma$ is a-balanced then $\tilde{\Delta}_{\prec}(\Gamma)$ is also a-balanced. Moreover, Babson and Novik proved that if $\Gamma$ is balanced and Cohen-Macaulay then $\tilde{\Delta}_{\prec}(\Gamma)$ is also Cohen-Macaulay for a certain order $\prec$ on $V$. On the other hand, since algebraic shifting does not change shifted complexes, it would be natural to ask whether the following property holds:

(C4) If $\Gamma$ is color-shifted then $\tilde{\Delta}_{\prec}(\Gamma)=\Gamma$.

In this paper, we prove this property (Corollary 1.11).

For a graded ideal $I \subset K[V]$, the integers $\beta_{i j}^{K[V]}(I)=\operatorname{dim}_{K} \operatorname{Tor}_{i}^{K[V]}(I, K)_{j}$ are called the graded Betti numbers of $I$. Since there are nice relations between algebraic 
shifting and graded Betti numbers (see [13]), it is also expected that there is a relation between the graded Betti numbers of $I_{\Gamma}$ and those of $I_{\tilde{\Delta}_{\prec}(\Gamma)}$. The main result of this paper is the following.

Theorem 0.1 Let $I \subset K[V]$ be a strongly color-stable ideal with $\operatorname{Gen}(I) \subset \mathcal{A}$. Then $\beta_{i j}^{K[V]}(I)=\beta_{i j}^{K[V]}(\tilde{\Phi}(I))$ for all $i$ and $j$.

The above theorem implies that the graded Betti numbers of $I_{\tilde{\Delta}_{<}(\Gamma)}$ are equal to those of the $G$-generic initial ideal of $I_{\Gamma}$. Thus, an immediate consequence of Theorem 0.1 is $\beta_{i j}^{K[V]}\left(I_{\Gamma}\right) \leq \beta_{i j}^{K[V]}\left(I_{\tilde{\Delta}_{<}(\Gamma)}\right)$ for all $i$ and $j$. Note that, in the case when $r=1$, Theorem 0.1 was shown in [4].

To prove Theorem 0.1, we use the exterior algebra and polarization (see Sect. 2). Let $I \subset K[V]$ be a strongly color-stable ideal and pol $(I)$ its polarization. Since all monomial ideals in the exterior algebra are squarefree monomial ideals, using the exterior algebra is sometimes useful for studying squarefree monomial ideals. Indeed, we find a nice relation between $\operatorname{pol}(I)$ and $\tilde{\Phi}(I)$ in terms of the exterior algebra. We show that, regarding $\operatorname{pol}(I)$ and $\tilde{\Phi}(I)$ as ideals in the exterior algebra, the $G$-generic initial ideal of $\operatorname{pol}(I)$ is equal to $\tilde{\Phi}(I)$ in the exterior algebra (by re-indexing the variables). Theorem 0.1 follows from this relation and property (C4) by using the relation between the graded Betti numbers of monomial ideals in the exterior algebra and those of monomial ideals in the polynomial ring, which was given by AramovaAvramov-Herzog [1].

Since algebraic shifting preserves the Betti numbers of simplicial complexes, it was expected that colored algebraic shifting preserves the Betti numbers of balanced complexes if we choose a certain order $\prec$ on $V$. However, we will give a counterexample to this problem in the last section of this paper.

This paper is organized as follows: In Sect. 1, we will recall colored algebraic shifting, and prove property (C4). In Sect. 2, we will study the relationship between polarization and generic initial ideals in the exterior algebra. The results in this section play a crucial role in the proof of Theorem 0.1 . The proof of Theorem 0.1 will be given in Sect. 3. In Sect. 4, we will show that colored algebraic shifting does not always preserve the Betti numbers of balanced complexes.

\section{Colored algebraic shifting}

In this section, we recall colored algebraic shifting defined by Babson and Novik [5]. Let $V=\bigcup_{j=1}^{r} V_{j}$ be a set of variables with $V_{j}=\left\{x_{j, 1}, x_{j, 2}, \ldots, x_{j, n_{j}}\right\}$. Set $G=$ $G L_{n_{1}}(K) \times G L_{n_{2}}(K) \times \cdots \times G L_{n_{r}}(K)$. Any $\varphi=\left(\varphi_{1}, \ldots, \varphi_{r}\right) \in G$ with each $\varphi_{j}=$ $\left(a_{s t}^{(j)}\right)_{1 \leq s, t \leq n_{j}} \in G L_{n_{j}}(K)$ defines the $\mathbb{Z}^{r}$-graded automorphism of $K[V]$ induced by $\varphi\left(x_{j, l}\right)=\sum_{k=1}^{n_{j}} a_{k l}^{(j)} x_{j, k}$. We say that a total order $\prec$ on $V$ is admissible if it satisfies $x_{j, 1} \succ x_{j, 2} \succ \cdots \succ x_{j, n_{j}}$ for all $j$. For a $\mathbb{Z}^{r}$-graded ideal $I \subset K[V]$, we write in $\prec(I)$ for the initial ideal of $I$ w.r.t. the reverse lexicographic order induced by $\prec$. The definition of colored algebraic shifting is based on the following generalization of generic initial ideals. 
Lemma 1.1 ([5, Theorem 5.3]) Let $I \subset K[V]$ be a $\mathbb{Z}^{r}$-graded ideal and $\prec$ a total order on $V$. There are nonempty Zariski open subsets $U_{1}, U_{2}, \ldots, U_{r}$ with each $U_{j} \subset$ $G L_{n_{j}}(K)$ such that $\operatorname{in}_{\prec} \varphi(I)$ is constant for all $\varphi \in U_{1} \times \cdots \times U_{r}$.

The above ideal $\operatorname{in}_{\prec} \varphi(I)$ with $\varphi \in U_{1} \times \cdots \times U_{r}$ is called the $G$-generic initial ideal of $I$ w.r.t. the admissible order $\prec$, and will be denoted $G$-gin $_{\prec}(I)$. Like generic initial ideals, $G$-generic initial ideals have a simpler structure.

Lemma 1.2 ([5, Theorem 5.4]) If $\operatorname{char}(K)=0$ then, for any $\mathbb{Z}^{r}$-graded ideal $I \subset$ $K[V]$ and for any admissible order $\prec, G$-gin $_{\prec}(I)$ is strongly color-stable.

Remark 1.3 In case of $r=1, G$-generic initial ideals are called generic initial ideals and strongly color-stable ideals are called strongly stable ideals. See e.g., [13]. Also, for $r=2, G$-generic initial ideals and strongly color-stable ideals are considered in [2].

Now we define colored algebraic shifting. Let $\Gamma$ be a simplicial complex on $V$ and $\prec$ an admissible order on $V$. Set $B=\left\{u \in \mathcal{M}[V]: u \notin G\right.$-gin $\left.{ }_{\prec}\left(I_{\Gamma}\right)\right\}$ and let $\mathcal{A}$ be the set of monomials defined in (1). The colored algebraic shifted complex $\tilde{\Delta}_{\prec}(\Gamma)$ of $\Gamma$ (w.r.t. $\prec$ ) is the collection of squarefree monomials defined by

$$
\tilde{\Delta}_{\prec}(\Gamma)=\{\tilde{\Phi}(u): u \in B \cap \mathcal{A}\} .
$$

It is not obvious that $\tilde{\Delta}_{\prec}(\Gamma)$ is a simplicial complex. However, it was proved in [5, Theorem 5.6] that it is indeed a simplicial complex and satisfies properties (C1)(C3). The map $\Gamma \rightarrow \tilde{\Delta}_{\prec}(\Gamma)$ is called colored algebraic shifting (w.r.t. $\left.\prec\right)$. In the rest of this section, we will study fundamental properties of colored algebraic shifting and the color-squarefree operation. First, we recall some results which appeared in [5, Lemma 5.2 and Theorem 5.6].

Lemma 1.4 (Babson and Novik) Let $\prec$ be an admissible order on $V$.

(i) The set $\mathcal{M}[V]$ of all monomials on $V$ is the set of the form

$$
\mathcal{M}[V]=\bigcup_{u \in \mathcal{A}}\left\{u w_{1} \cdots w_{r}: w_{j} \in \mathcal{M}\left[x_{j, n_{j}+1-\operatorname{Deg}_{j}(u)}, \ldots, x_{j, n_{j}}\right] \text { for each } j\right\} .
$$

(ii) Let $\Gamma$ be a simplicial complex on $V$ and $B=\left\{u \in \mathcal{M}[V]: u \notin G\right.$-gin (I $\left.\left._{\Gamma}\right)\right\}$. If $\operatorname{char}(K)=0$ then $B$ is the multicomplex of the form

$$
B=\bigcup_{u \in B \cap \mathcal{A}}\left\{u w_{1} \cdots w_{r}: w_{j} \in \mathcal{M}\left[x_{j, n_{j}+1-\operatorname{Deg}_{j}(u)}, \ldots, x_{j, n_{j}}\right] \forall j\right\} .
$$

(iii) Let I be a strongly color-stable ideal in $K[V], B=\{u \in \mathcal{M}[V]: u \notin I\}$ and $\Gamma=$ $\{\tilde{\Phi}(u): u \in B \cap \mathcal{A}\}$. If $B$ is a multicomplex of the form (2), then $\Gamma$ is a simplicial complex and $I_{\Gamma}$ has the same Hilbert function as $I$, that is, $\operatorname{dim}_{K}\left(I_{\Gamma}\right)_{t}=\operatorname{dim}_{K} I_{t}$ for all $t$.

Note that (ii) and (iii) imply that $\tilde{\Delta}_{\prec}(\Gamma)$ is a simplicial complex for any simplicial complex $\Gamma$ on $V$. 
Lemma 1.5 Let $I \subset K[V]$ be a strongly color-stable monomial ideal and $B=\{u \in$ $\mathcal{M}[V]: u \notin I\}$. The following conditions are equivalent.

(i) $\operatorname{Gen}(I) \subset \mathcal{A}$;

(ii) $B$ is a multicomplex of the form (2).

Proof ((i) $\Rightarrow$ (ii)) Let $u=u_{1} u_{2} \cdots u_{r} \in \mathcal{A}$ where each $u_{j} \in K\left[V_{j}\right]$, and $w_{j} \in$ $\mathcal{M}\left[x_{j, n_{j}+1-\operatorname{Deg}_{j}(u)}, \ldots, x_{j, n_{j}}\right]$ for $j=1,2, \ldots, r$. By Lemma 1.4 (i) it is enough to show that $u \in B$ if and only if $u w_{1} \cdots w_{r} \in B$. The 'if' part immediately follows since $B$ is a multicomplex. We will show the 'only if' part.

Suppose $u w_{1} \cdots w_{r} \notin B$. We must prove that $u \notin B$, that is, $u \in I$. Since $u w_{1} \cdots w_{r} \in I$, there exists $v=v_{1} \cdots v_{r} \in \operatorname{Gen}(I)$ with $v_{j} \in K\left[V_{j}\right]$ such that $v$ divides $u w_{1} \cdots w_{r}$. Notice that each $v_{j} \in \mathcal{A}$ by assumption. Since $I$ is strongly colorstable, we may assume that $v_{j} \leq_{\mathrm{P}} u_{j} w_{j}$. Let $v_{j}=x_{j, p_{1}} \cdots x_{j, p_{k}}, u_{j}=x_{j, q_{1}} \cdots x_{j, q_{\ell}}$ and $w_{j}=x_{j, q_{\ell+1}} \cdots x_{j, q_{\ell+s}}$, where $p_{1} \leq \cdots \leq p_{k}, q_{1} \leq \cdots \leq q_{\ell}$ and $q_{\ell+1} \leq \cdots \leq$ $q_{\ell+s}$. Since $u_{j} \in \mathcal{A}$ we have $q_{\ell} \leq q_{\ell+1}$. Also, since $v_{j}$ divides $u_{j} w_{j}$ and $v_{j} \leq{ }_{P}$ $u_{j} w_{j}$, we have $p_{i}=q_{i}$ for $i=1,2, \ldots, k$. Thus $v_{j}$ divides $u_{j}$ if $k \leq \ell$ and $u_{j}$ divides $v_{j}$ if $k>\ell$. On the other hand, by Lemma 1.4 (i), for any monomial $w \in K\left[x_{j, n_{j}+1-\operatorname{Deg}_{j}(u)}, \ldots, x_{j, n_{j}}\right]$, we have $u_{j} w \notin \mathcal{A}$ if $w \neq 1$. Thus if $u_{j}$ divides $v_{j}$ then $u_{j}=v_{j}$ since $v_{j} \in \mathcal{A}$. Hence $v_{j}$ divides $u_{j}$ for $j=1,2, \ldots, r$. Since $v=v_{1} \cdots v_{r} \in I$, we have $u=u_{1} \cdots u_{r} \in I$ as desired.

$((\mathrm{ii}) \Rightarrow(\mathrm{i}))$ Let $v \in \operatorname{Gen}(I)$. Then Lemma 1.4 (i) says that there exists a monomial $u \in \mathcal{A}$ such that $v \in\left\{u w_{1} \cdots w_{r}: w_{j} \in \mathcal{M}\left[x_{j, n_{j}+1-\operatorname{Deg}_{j}(u)}, \ldots, x_{j, n_{j}}\right]\right.$ for each $\left.j\right\}$. Since $v \in I$ the assumption says that $u \in I$. Since $v \in \operatorname{Gen}(I)$ and $u$ divides $v$, we have $v=u \in \mathcal{A}$ as desired.

If $I \subset K[V]$ is a monomial ideal satisfying $\operatorname{Gen}(I) \subset \mathcal{A}$, then we write $\tilde{\Phi}(I)$ for the squarefree monomial ideal in $K[V]$ generated by $\{\tilde{\Phi}(u): u \in \operatorname{Gen}(I)\}$. Similarly for any squarefree monomial ideal $J \subset K[V]$, write $\tilde{\Phi}^{-1}(J)$ for the monomial ideal generated by $\left\{\tilde{\Phi}^{-1}(u): u \in \operatorname{Gen}(J)\right\}$. The following result gives another definition of colored algebraic shifting.

Corollary 1.6 Let $\Gamma$ be a simplicial complex on $V$ and $\prec$ an admissible order. Then $\operatorname{Gen}\left(G-\operatorname{gin}_{\prec}\left(I_{\Gamma}\right)\right) \subset \mathcal{A}$ and $I_{\tilde{\Delta}_{\prec}(\Gamma)}=\tilde{\Phi}\left(G-\operatorname{gin}_{\prec}\left(I_{\Gamma}\right)\right)$.

Proof The first statement follows from Lemma 1.4 (ii) and Lemma 1.5. We will consider the second statement. By the definition of $\tilde{\Delta}_{\prec}(\Gamma)$, we have $u \in G$-gin ${ }_{\prec}\left(I_{\Gamma}\right) \cap \mathcal{A}$ if and only if $\tilde{\Phi}(u) \notin \tilde{\Delta}_{\prec}(\Gamma)$. Hence $u \in \operatorname{Gen}\left(G\right.$-gin $\left.\operatorname{gin}_{\prec}\left(I_{\Gamma}\right)\right)$ implies $\tilde{\Phi}(u) \in I_{\tilde{\Delta}_{\prec}(\Gamma)}$, and $v \in \operatorname{Gen}\left(I_{\tilde{\Delta}_{\prec}(\Gamma)}\right)$ implies $\tilde{\Phi}^{-1}(v) \in G$-gin $\operatorname{ri}_{\prec}\left(I_{\Gamma}\right)$ and $v \in \tilde{\Phi}\left(G\right.$-gin gin $\left._{\prec}\left(I_{\Gamma}\right)\right)$ (see [16, Lemma 1.7]). This fact says that $I_{\tilde{\Delta}_{\prec}(\Gamma)} \supset \tilde{\Phi}\left(G-\operatorname{gin}{ }_{\prec}\left(I_{\Gamma}\right)\right)$ and $I_{\tilde{\Delta}_{\prec}(\Gamma)} \subset$ $\tilde{\Phi}\left(G\right.$-gin $\left.\left.\operatorname{si}_{\Gamma}\right)\right)$ as desired.

Next we list some fundamental properties of $\tilde{\Phi}$. A squarefree monomial ideal $I \subset$ $K[V]$ is said to be squarefree strongly color-stable if, for all squarefree monomials $u \in I$ and $v \leq_{\mathrm{P}} u$ with $\operatorname{Deg}(v)=\operatorname{Deg}(u)$, it follows that $v \in I$. 
Lemma 1.7 Let I and $J$ be strongly color-stable ideals in $K[V]$ satisfying $\operatorname{Gen}(I) \subset$ $\mathcal{A}$ and $\operatorname{Gen}(J) \subset \mathcal{A}$. Set $\Gamma=\{\tilde{\Phi}(u): u \notin I$ and $u \in \mathcal{A}\}$. Then

(a) If $u \in I \cap \mathcal{A}$ then $\tilde{\Phi}(u) \in \tilde{\Phi}(I)$.

(b) If $u \in \tilde{\Phi}(I)$ is a squarefree monomial then $\tilde{\Phi}^{-1}(u) \in I \cap \mathcal{A}$.

(c) I and $\tilde{\Phi}(I)$ have the same Hilbert function.

(d) $\tilde{\Phi}(I)$ is a squarefree strongly color-stable ideal.

(e) If $I^{\prime}$ is a squarefree strongly color-stable ideal in $K[V]$ then $\tilde{\Phi}^{-1}\left(I^{\prime}\right)$ is strongly color-stable.

(f) One has $I \subset J$ if and only if $\tilde{\Phi}(I) \subset \tilde{\Phi}(J)$.

(g) If $u$ and $v$ are monomials satisfying $u \in \operatorname{Gen}(I), v \leq_{\mathrm{P}} u$ and $\operatorname{Deg}(v)=\operatorname{Deg}(u)$ then $\tilde{\Phi}(v) \in \tilde{\Phi}(I)$.

Proof ((a), (b) and (c)) Lemmas 1.4 (iii) and 1.5 say that $\Gamma$ is a simplicial complex and $I_{\Gamma}$ has the same Hilbert function as $I$. On the other hand we have $\tilde{\Phi}(I)=I_{\Gamma}$ in the same way as in Corollary 1.6. Then statements follow from the definition of $\Gamma$.

(d) It suffices to show that if $u \in \operatorname{Gen}(\tilde{\Phi}(I))$ and $v$ is a squarefree monomial satisfying $v \leq_{\mathrm{P}} u$ and $\operatorname{Deg}(v)=\operatorname{Deg}(u)$ then $v \in \tilde{\Phi}(I)$. Since $v \leq_{\mathrm{P}} u, \operatorname{Deg}(v)=\operatorname{Deg}(u)$ and $\tilde{\Phi}^{-1}(u) \in \mathcal{A}$, it follows that $\tilde{\Phi}^{-1}(v) \leq_{\mathrm{P}} \tilde{\Phi}^{-1}(u)$ and $\tilde{\Phi}^{-1}(v) \in \mathcal{A}$. Also, since $I$ is strongly color-stable, we have $\Phi^{-1}(v) \in I \cap \mathcal{A}$. Then statement (a) implies $v \in \tilde{\Phi}(I)$.

(e) This can be proved in the same way as (d).

(f) Since $\operatorname{Gen}(I) \subset \mathcal{A}$ and $\operatorname{Gen}(J) \subset \mathcal{A}$ the statement follows from (a) and (b).

(g) Since $I$ is strongly color-stable, this is a special case of (a).

Next, we will show that $\tilde{\Delta}_{\prec}(\Gamma)=\Gamma$ if $\Gamma$ is color-shifted. Recall that if $r=1$ then $G$-generic initial ideals are generic initial ideals and colored algebraic shifting is called symmetric algebraic shifting. In this special case, the next fact was known (see [4, Corollary 1.6] and [16, Theorem 1.6]).

Lemma 1.8 Fix $1 \leq j \leq r$. Let I be a strongly colored-stable ideal in $K\left[V_{j}\right]$ with $\operatorname{Gen}(I) \subset \mathcal{A}$ and $\prec$ an admissible order on $V$. Then there exists a nonempty Zariski open subset $U \subset G L_{n_{j}}(K)$ such that $\operatorname{in}_{\prec} \varphi(\tilde{\Phi}(I))=I$ for all $\varphi \in U$.

Corollary 1.9 Let $u \in \mathcal{M}\left[V_{j}\right] \cap \mathcal{A}, \mathcal{G}=\left\{v \in \mathcal{M}\left[V_{j}\right]: v \leq_{\mathrm{P}} u\right.$ and $\left.\operatorname{deg}(v)=\operatorname{deg}(u)\right\}$ and $\prec$ an admissible order on $V$. Then there exists a nonempty Zariski open subset $U \subset G L_{n_{j}}(K)$ such that, for each $\varphi \in U$, there exist monomials $v_{1}, \ldots, v_{k}$ in $\mathcal{G}$ and elements $a_{1}, \ldots, a_{k}$ of $K$ such that

$$
\operatorname{in}_{\prec} \varphi\left(a_{1} \tilde{\Phi}\left(v_{1}\right)+a_{2} \tilde{\Phi}\left(v_{2}\right)+\cdots+a_{k} \tilde{\Phi}\left(v_{k}\right)\right)=u \text {. }
$$

Proof Let $I$ be the monomial ideal in $K\left[V_{j}\right]$ generated by $\mathcal{G}$. Then $I$ is strongly color-stable and $\operatorname{Gen}(I) \subset \mathcal{A}$. Thus Lemma 1.8 says that there exists a nonempty Zariski open subset $U \subset G L_{n_{j}}(K)$ such that $\operatorname{in}_{\prec} \varphi(\tilde{\Phi}(I))=I$ for any $\varphi \in U$. Then, for each $\varphi \in U$, there are monomials $v_{1}^{\prime}, v_{2}^{\prime}, \ldots, v_{k}^{\prime}$ of degree $\operatorname{deg}(u)$ in $\tilde{\Phi}(I)$ and elements $a_{1}, a_{2}, \ldots, a_{k}$ of $K$ such that

$$
\operatorname{in}_{\prec}\left(\varphi\left(a_{1} v_{1}^{\prime}+a_{2} v_{2}^{\prime}+\cdots+a_{k} v_{k}^{\prime}\right)\right)=u \text {. }
$$

On the other hand, by the definition of $I$, the set of all monomials of degree $\operatorname{deg}(u)$ in $\tilde{\Phi}(I)$ is $\{\tilde{\Phi}(v): v \in \mathcal{G}\}$. Hence $v_{t}^{\prime} \in\{\tilde{\Phi}(v): v \in \mathcal{G}\}$ for $t=1,2, \ldots, k$. 
Theorem 1.10 Let $I \subset K[V]$ be a strongly color-stable ideal with $\operatorname{Gen}(I) \subset \mathcal{A}$. Then, for any admissible order $\prec$ on $V$, one has $G-\operatorname{gin}_{\prec}(\tilde{\Phi}(I))=I$.

Proof Since Lemma 1.7 says that $I$ and $G$-gin $\sin _{\precsim}(\tilde{\Phi}(I))$ have the same Hilbert function, it suffices to show that $\operatorname{Gen}(I) \subset G$-gin gin $_{\prec}(\tilde{\Phi}(I))$.

Let $u=u_{1} u_{2} \cdots u_{r} \in \operatorname{Gen}(I)$ where each $u_{j} \in \mathcal{M}\left[V_{j}\right]$. We will show that $u \in$ $G$-gin g $_{\prec}(\tilde{\Phi}(I))$. By Lemma 1.1 and Corollary 1.9 , there exists a $\varphi=\left(\varphi_{1}, \ldots, \varphi_{r}\right) \in$ $G$ with each $\varphi_{j} \in G L_{n_{j}}(K)$ such that $\operatorname{in}_{\prec} \varphi(\tilde{\Phi}(I))=G-\operatorname{gin}{ }_{\prec}(\tilde{\Phi}(I))$ and, for $j=1,2, \ldots, r$, there exist monomials $v_{j, 1}, \ldots, v_{j, k_{j}} \in\left\{v \in \mathcal{M}\left[V_{j}\right]: v \leq_{\mathrm{P}} u_{j}\right.$ and $\left.\operatorname{deg}(v)=\operatorname{deg}\left(u_{j}\right)\right\}$ and elements $a_{j, 1}, \ldots, a_{j, k_{j}} \in K$, such that

$$
\operatorname{in}_{\prec}\left(\varphi_{j}\left\{a_{j, 1} \tilde{\Phi}\left(v_{j, 1}\right)+\cdots+a_{j, k_{j}} \tilde{\Phi}\left(v_{j, k_{j}}\right)\right\}\right)=u_{j} .
$$

Set

$$
f_{j}=a_{j, 1} \tilde{\Phi}\left(v_{j, 1}\right)+\cdots+a_{j, k_{j}} \tilde{\Phi}\left(v_{j, k_{j}}\right) \in K\left[V_{j}\right] .
$$

Since $\operatorname{in}_{\prec} \varphi_{j}\left(f_{j}\right)=u_{j}$, we have

$$
\operatorname{in}_{\prec} \varphi\left(f_{1} f_{2} \cdots f_{r}\right)=\left\{\operatorname{in}_{\prec} \varphi_{1}\left(f_{1}\right)\right\}\left\{\operatorname{in}_{\prec} \varphi_{2}\left(f_{2}\right)\right\} \cdots\left\{\operatorname{in}_{\prec} \varphi_{r}\left(f_{r}\right)\right\}=u_{1} u_{2} \cdots u_{r} .
$$

On the other hand, $f_{1} f_{2} \cdots f_{r}$ is a linear combination of monomials in $\mathcal{G}=\{\tilde{\Phi}(v)$ : $v \leq_{\mathrm{P}} u$ and $\left.\operatorname{Deg}(v)=\operatorname{Deg}(u)\right\}$. Since Lemma $1.7(\mathrm{~g})$ says that $\mathcal{G} \subset \tilde{\Phi}(I)$, it follows that $f_{1} f_{2} \cdots f_{r} \in \tilde{\Phi}(I)$ and $u=u_{1} u_{2} \cdots u_{r} \in \operatorname{in}_{\prec} \varphi(\tilde{\Phi}(I))=G$-gin $\prec(\tilde{\Phi}(I))$. Then we have $\operatorname{Gen}(I) \subset G$-gin gin $_{(}(\tilde{\Phi}(I))$ as desired.

Corollary 1.11 If $\Gamma$ is a color-shifted simplicial complex on $V$ then $\tilde{\Delta}_{\prec}(\Gamma)=\Gamma$ for any admissible order $\prec$ on $V$.

Proof Clearly $I_{\Gamma}$ is squarefree strongly color-stable. Thus Lemma 1.7 (e) says that $\tilde{\Phi}^{-1}\left(I_{\Gamma}\right)$ is strongly color-stable. Then Theorem 1.10 says that $G$-gin ${ }_{\prec}\left(I_{\Gamma}\right)=$ $\tilde{\Phi}^{-1}\left(I_{\Gamma}\right)$ and Corollary 1.6 says that $I_{\tilde{\Delta}_{\prec}(\Gamma)}=\tilde{\Phi}\left(G-\operatorname{gin}_{\prec}\left(I_{\Gamma}\right)\right)=I_{\Gamma}$.

\section{Polarization and squarefree stable operators}

First, we recall the notion of polarization of monomial ideals. Let $\Lambda$ be a set of indices, $X=\left\{x_{\tau}: \tau \in \Lambda\right\}$ a set of variables and $K[X]$ the polynomial ring over a field $K$ in the set of variables $X$. Consider the set of variables $\tilde{X}=\left\{x_{\tau,[k]}: x_{\tau} \in\right.$ $\left.X, k \in \mathbb{Z}_{>0}\right\}$. Define the map pol $: \mathcal{M}[X] \rightarrow \mathcal{M}[\tilde{X}]$ by

$$
\operatorname{pol}\left(x_{\tau_{1}}^{a_{1}} x_{\tau_{2}}^{a_{2}} \ldots x_{\tau_{k}}^{a_{k}}\right)=\prod_{j=1}^{k}\left(x_{\tau_{j},[1]} x_{\tau_{j},[2]} \cdots x_{\tau_{j},\left[a_{j}\right]}\right)
$$

where each $\tau_{t} \in \Lambda$. For any monomial ideal $I \subset K[X]$, write $\operatorname{pol}(I) \subset K[\tilde{X}]$ for the monomial ideal generated by $\{\operatorname{pol}(u): u \in \operatorname{Gen}(I)\}$. The ideal $\operatorname{pol}(I)$ is called the polarization of $I$. Note that $\operatorname{pol}(I)$ is always a squarefree monomial ideal. 
There is a nice relationship between polarization and graded Betti numbers. For a finitely generated graded ideal $I \subset K[X]$, we define the graded Betti numbers of $I$ by $\beta_{i j}^{K[X]}(I)=\operatorname{dim}_{K} \operatorname{Tor}_{i}^{K\left[X^{\prime}\right]}\left(I \cap K\left[X^{\prime}\right], K\right)_{j}$ where $X^{\prime} \subset X$ is a finite subset satisfying $\operatorname{Gen}(I) \subset K\left[X^{\prime}\right]$. Note that these numbers are independent of the choice of $X^{\prime}$ with $\operatorname{Gen}(I) \subset K\left[X^{\prime}\right]$. The following facts are known.

\section{Lemma 2.1 Let I be a finitely generated monomial ideal in $K[X]$.}

(i) I and its polarization pol(I) have the same graded Betti numbers, that is, $\beta_{i j}^{K[X]}(I)=\beta_{i j}^{K[\tilde{X}]}(\operatorname{pol}(I))$ for all $i$ and $j$

(ii) If $I \subset J$ are monomial ideals in $K[X]$ then $\operatorname{pol}(I) \subset \operatorname{pol}(J)$.

See [10, Lemma 4.16] for the proof of statement (i). Also, statement (ii) follows from the fact that if $u, v \in \mathcal{M}[X]$ and $u$ divides $v$ then $\operatorname{pol}(u)$ divides $\operatorname{pol}(v)$.

The following nice fact is known: Let $I$ be a monomial ideal of $K\left[x_{1,1}, \ldots, x_{1, n_{1}}\right]$. Suppose that $n_{1}$ is sufficiently large. Then we may assume that $\operatorname{pol}(I)$ is an ideal of $K\left[x_{1,1}, \ldots, x_{1, n_{1}}\right]$. It was proved in [6] that if $I$ is strongly stable (see Remark 1.3) then the generic initial ideal of $\operatorname{pol}(I)$ with respect to the reverse lexicographic order is equal to $I$.

The aim of this section is to give an analogue of the above fact for an exterior algebra. Let, as before, $V=\dot{U}_{j=1}^{r} V_{j}$ be a set of variables with $V_{j}=\left\{x_{j, 1}, \ldots, x_{j, n_{j}}\right\}$ for $j=1, \ldots, r$, and let $\bigwedge\langle V\rangle$ be the exterior algebra over a field $K$ in the set of variables $V$. Let $\mathcal{N}\langle V\rangle$ be the set of monomials in $\bigwedge\langle V\rangle$, where a monomial of $\bigwedge\langle V\rangle$ is an element of $\bigwedge\langle V\rangle$ of the form

$$
x_{i_{1}, j_{1}} \wedge x_{i_{2}, j_{2}} \wedge \cdots \wedge x_{i_{p}, j_{p}}
$$

where $i_{1} \leq i_{2} \leq \cdots \leq i_{p}$ and where $j_{t}<j_{t+1}$ if $i_{t}=i_{t+1}$. Define the $\mathbb{Z}^{r}$-grading of $\bigwedge\langle V\rangle$ in the same way as for the polynomial ring $K[V]$. For an admissible order $\prec$ and for a $\mathbb{Z}^{r}$-graded ideal $J \subset \bigwedge\langle V\rangle$, write in $_{\prec} J$ for the initial ideal of $J$ w.r.t. the reverse lexicographic order induced by $\prec$. We refer the reader to [3] for foundations on the Gröbener basis theory in exterior algebras.

For each monomial $u=x_{i_{1}, j_{1}} \wedge x_{i_{2}, j_{2}} \wedge \cdots \wedge x_{i_{p}, j_{p}} \in \bigwedge\langle V\rangle$, set

$$
u^{\natural}=x_{i_{1}, j_{1}} x_{i_{2}, j_{2}} \cdots x_{i_{p}, j_{p}} \in K[V] .
$$

Similarly, for a squarefree monomial $v=x_{i_{1}, j_{1}} \cdots x_{i_{p}, j_{p}} \in \mathcal{M}[V]$, where $i_{1} \leq \cdots \leq$ $i_{p}$ and $j_{t}<j_{t+1}$ if $i_{t}=i_{t+1}$ for all $t$, we write $v^{b}=x_{i_{1}, j_{1}} \wedge \cdots \wedge x_{i_{p}, j_{p}} \in \wedge\langle V\rangle$ (this $v^{b}$ is well-defined by the ordering of the variables). For a monomial ideal $J \subset \bigwedge\langle V\rangle$, let $J^{\natural}$ be the monomial ideal in $K[V]$ generated by $\left\{u^{\natural}: u \in \operatorname{Gen}(J)\right\}$. Also, for a squarefree monomial ideal $I \subset K[V]$, define $I^{\mathrm{b}} \subset \bigwedge\langle V\rangle$ similarly. We say that a monomial ideal $J \subset \bigwedge\langle V\rangle$ is squarefree strongly color-stable if $J^{\natural}$ is.

Definition 2.2 To simplify, set $n_{1}=n$ and $x_{1, t}=x_{t}$ for all $t$. Hence $V_{1}=$ $\left\{x_{1}, \ldots, x_{n}\right\}$. Let $W \supset V_{1}$ be the set of infinitely many variables $x_{1}, x_{2}, \ldots$ We define $^{\natural}$ and ${ }^{b}$ on $K[W]$ and $\bigwedge\langle W\rangle$ in the same way as for $K[V]$ and $\bigwedge\langle V\rangle$. Extend the partial order $<_{\mathrm{P}}$ on $K\left[V_{1}\right]$ to $K[W]$. A monomial ideal $I$ in $K[W]$ (or in 
$\bigwedge\langle W\rangle)$ is called squarefree strongly stable if, for all squarefree monomials $u \in I$ and $v<_{\mathrm{P}} u$ with $\operatorname{deg}(v)=\operatorname{deg}(u)$, it follows that $v \in I$. A squarefree stable operator $\sigma: \mathcal{N}\langle W\rangle \rightarrow \mathcal{N}\langle W\rangle$ is a map that satisfies

(i) if $J \subset \bigwedge\langle W\rangle$ is a finitely generated squarefree strongly stable ideal then $J^{\natural}$ and $\sigma(J)^{\natural}$ have the same graded Betti numbers, where $\sigma(J)$ is the monomial ideal generated by $\{\sigma(u): u \in \operatorname{Gen}(J)\}$;

(ii) if $J \subset J^{\prime}$ are finitely generated strongly stable monomial ideals in $\bigwedge\langle W\rangle$ then $\sigma(J) \subset \sigma\left(J^{\prime}\right)$.

If $J$ is a finitely generated graded ideal in $\bigwedge\left\langle V_{1}\right\rangle$ or in $\bigwedge\langle W\rangle$ then we write $\operatorname{in}(J)$ for the initial ideal of $J$ w.r.t. the reverse lexicographic order induced by $x_{1}>x_{2}>\cdots$. The significance of squarefree stable operators is explained by the following statement.

Lemma 2.3 ([16, Proposition 7.4]) Let $\sigma: \mathcal{N}\langle W\rangle \rightarrow \mathcal{N}\langle W\rangle$ be a squarefree stable operator and $J \subset \wedge\left\langle V_{1}\right\rangle$ a squarefree strongly stable ideal satisfying $\{\sigma(u)$ : $u \in \operatorname{Gen}(J)\} \subset \bigwedge\left\langle V_{1}\right\rangle$. Let $\sigma(J)$ be the ideal in $\bigwedge\left\langle V_{1}\right\rangle$ generated by $\{\sigma(u): u \in$ $\operatorname{Gen}(J)\}$. Then there exists a nonempty Zariski open subset $U \subset G L_{n}(K)$ such that $\operatorname{in}(\varphi(\sigma(J)))=J$ for all $\varphi \in U$.

We will define a new squarefree stable operator by using polarization. Recall that the squarefree operation $\Phi: \mathcal{M}[W] \rightarrow \mathcal{M}[W]$ is the map defined by $\Phi\left(x_{i_{1}} x_{i_{2}} \cdots x_{i_{k}}\right)=x_{i_{1}} x_{i_{2}+1} \cdots x_{i_{k}+k-1}$ where $i_{1} \leq i_{2} \leq \cdots \leq i_{k}$. Hence this is a special case of the color-squarefree operation and we have $\Phi(u)=\tilde{\Phi}(u)$ if $u \in$ $\mathcal{M}\left[V_{1}\right] \cap \mathcal{A}$. The following fact is known.

Lemma 2.4 ([4, Lemma 2.2]) If $I \subset K[W]$ is a finitely generated strongly stable ideal then $I$ and $\Phi(I)$ have the same graded Betti numbers. by

Let pol $^{*}: \mathcal{N}\langle W\rangle \rightarrow \mathcal{N}\langle\tilde{W}\rangle$, where $\tilde{W}=\left\{x_{i,[k]}: i \geq 1, k \geq 1\right\}$, be the map defined

$$
\operatorname{pol}^{*}(u)=\operatorname{pol}\left(\Phi^{-1}\left(u^{\natural}\right)\right)^{b} \quad \text { for any } u \in \mathcal{N}\langle W\rangle \text {. }
$$

The next fact easily follows from Lemmas 2.1 and 2.4.

Lemma 2.5 Let $J$ and $J^{\prime}$ be finitely generated squarefree strongly stable ideals in $\bigwedge\langle W\rangle$. Then

(i) $J^{\natural}$ and $\left(\mathrm{pol}^{*}(J)\right)^{\natural}$ have the same graded Betti numbers;

(ii) if $J \subset J^{\prime}$ then $\operatorname{pol}^{*}(J) \subset \operatorname{pol}^{*}\left(J^{\prime}\right)$.

Proof Clearly $\left(\operatorname{pol}^{*}(J)\right)^{\natural}=\operatorname{pol}\left(\Phi^{-1}\left(J^{\natural}\right)\right) \subset K[\tilde{W}]$. Since $\Phi$ is a special case of the color-squarefree operation, Lemma 1.7 (e) says that $\Phi^{-1}\left(J^{\natural}\right)$ is strongly stable. Then Lemma 2.4 says that $J^{\natural}$ and $\Phi^{-1}\left(J^{\natural}\right)$ have the same graded Betti numbers. Hence $J^{\natural}$ and $\left(\operatorname{pol}^{*}(J)\right)^{\natural}$ have the same graded Betti numbers by Lemma 2.1 (i).

If $J \subset J^{\prime}$ then $\Phi^{-1}\left(J^{\natural}\right) \subset \Phi^{-1}\left(\left(J^{\prime}\right)^{\natural}\right)$ by Corollary 1.7 (f). Then we have $\operatorname{pol}^{*}(J) \subset \operatorname{pol}^{*}\left(J^{\prime}\right)$ by Lemma 2.1 (ii). 
Example 2.6 Let $J=\left(x_{1} \wedge x_{2} \wedge x_{3}, x_{1} \wedge x_{2} \wedge x_{4}\right)$. Then

$$
\begin{aligned}
\operatorname{pol}^{*}(J) & =\left(\operatorname{pol}\left(\Phi^{-1}\left(x_{1} x_{2} x_{3}\right)\right), \operatorname{pol}\left(\Phi^{-1}\left(x_{1} x_{2} x_{4}\right)\right)\right)^{b} \\
& =\left(\operatorname{pol}\left(x_{1}^{3}\right), \operatorname{pol}\left(x_{1}^{2} x_{2}\right)\right)^{b} \\
& =\left(x_{1,[1]} \wedge x_{1,[2]} \wedge x_{1,[3]}, x_{1,[1]} \wedge x_{1,[2]} \wedge x_{2,[1]}\right)
\end{aligned}
$$

Fix a bijection $\pi: \mathbb{Z}_{>0} \times \mathbb{Z}_{>0} \rightarrow \mathbb{Z}_{>0}$. Then $\pi$ induces an isomorphism of $\mathbb{Z}$ graded $K$-algebras from $\bigwedge\langle\tilde{W}\rangle$ to $\bigwedge\langle W\rangle$ by setting $\pi\left(x_{i,[k]}\right)=x_{\pi(i, k)}$. Let $\Psi$ : $\mathcal{N}\langle W\rangle \rightarrow \mathcal{N}\langle W\rangle$ be the map defined by $\Psi=\pi \circ$ pol $^{*}$. Then, by Lemma 2.5 , we have

Proposition 2.7 The map $\Psi: \mathcal{N}\langle W\rangle \rightarrow \mathcal{N}\langle W\rangle$ is a squarefree stable operator.

Corollary 2.8 Let $u \in \mathcal{N}\left\langle V_{1}\right\rangle$ and $\mathcal{G}=\left\{v \in \mathcal{N}\left\langle V_{1}\right\rangle: v \leq_{\mathrm{P}} u\right.$ and $\left.\operatorname{deg}(v)=\operatorname{deg}(u)\right\}$. Assume that $\Psi(v) \in \mathcal{N}\left\langle V_{1}\right\rangle$ for all $v \in \mathcal{G}$. Then, there exists a nonempty Zariski open subset $U \subset G L_{n_{1}}(K)$ which satisfies that, for each $\varphi \in U$, there are monomials $v_{1}, \ldots, v_{k}$ of $\mathcal{G}$ and elements $a_{1}, \ldots, a_{k}$ of $K$ such that

$$
\operatorname{in} \varphi\left(a_{1} \Psi\left(v_{1}\right)+\cdots+a_{k} \Psi\left(v_{k}\right)\right)=u .
$$

Proof Let $J \subset \bigwedge\left\langle V_{1}\right\rangle$ be the squarefree strongly stable ideal generated by $\mathcal{G}$. Then, since $\Psi$ is a squarefree stable operator, Lemma 2.3 says that there exists a nonempty Zariski open subset $U \subset G L_{n_{1}}(K)$ such that $\operatorname{in} \varphi(\Psi(J))=J$ for any $\varphi \in U$. Then the claim follows in the same way as Corollary 1.9.

Now, we return to the case $V=\bigcup_{j=1}^{r} V_{j}$ with $V_{j}=\left\{x_{j, 1}, \ldots, x_{j, n_{j}}\right\}$. Set $\tilde{V}=$ $\left\{x_{s, t,[k]}: x_{s, t} \in V, k \geq 1\right\}$. Consider infinitely many variables $x_{j, t}$ with $1 \leq j \leq r$ and $t \geq 1$, and set $\pi\left(x_{j, t,[k]}\right)=x_{j, \pi(t, k)}$. For any monomial $u \in \mathcal{N}\langle V\rangle$, define

$$
\tilde{\Psi}(u)=\left(\pi \circ \operatorname{pol} \circ \tilde{\Phi}^{-1}\left(u^{\natural}\right)\right)^{b} .
$$

The next statement, which is an analogue of Theorem 1.10, plays an important role in the proof of the main theorem of the next section.

Proposition 2.9 Let $J \subset \bigwedge\langle V\rangle$ be a squarefree strongly color-stable ideal, $\mathcal{G}=\{v \in$ $\mathcal{N}\langle V\rangle: v \leq_{\mathrm{P}} u$ and $\operatorname{Deg}(v)=\operatorname{Deg}(u)$ for some $\left.u \in \operatorname{Gen}(J)\right\}$ and $\prec$ an admissible order on $V$. Assume that $\tilde{\Psi}(v) \in \mathcal{N}\langle V\rangle$ for all $v \in \mathcal{G}$. Then there exists a $\varphi \in G=$ $G L_{n_{1}}(K) \times \cdots \times G L_{n_{r}}(K)$ such that $\operatorname{in}_{\prec} \varphi(\tilde{\Psi}(J))=J$, where $\tilde{\Psi}(J)$ is the ideal in $\bigwedge\langle V\rangle$ generated by $\{\tilde{\Psi}(u): u \in \operatorname{Gen}(J)\} \subset \bigwedge\langle V\rangle$.

Proof The idea of the proof is essentially the same as that of Theorem 1.10. Recall that if graded ideals have the same graded Betti numbers then they have the same Hilbert functions. Since $\tilde{\Psi}(J)=\pi\left(\operatorname{pol}\left(\tilde{\Phi}^{-1}\left(J^{\natural}\right)\right)\right)^{b}$, Lemmas 1.7 (c) and 2.1 say that $J$ and $\tilde{\Psi}(J)$ have the same Hilbert function. Thus it is enough to show that there exists $\varphi \in G$ such that $\operatorname{Gen}(J) \subset \operatorname{in}_{\prec} \varphi(\tilde{\Psi}(J))$. 
For any $u=u_{1} \cdots u_{r} \in \operatorname{Gen}(J)$ where each $u_{j} \in \mathcal{N}\left\langle V_{j}\right\rangle$, Corollary 2.8 says that there exists a nonempty Zariski open subset $U_{j, u} \subset G L_{n_{j}}(K)$ with the property that, for any $\varphi_{j} \in U_{j, u}$, there are monomials $v_{j, 1}, \ldots, v_{j, k_{j}}$ satisfying $\operatorname{Deg}\left(v_{j, t}\right)=$ $\operatorname{Deg}\left(u_{j}\right)$ and $v_{j, t} \leq_{\mathrm{P}} u_{j}$ for all $t$, and elements $a_{j, 1}, \ldots, a_{j, k_{j}}$ of $K$ such that

$$
\operatorname{in}_{\prec} \varphi_{j}\left\{a_{j, 1} \tilde{\Psi}\left(v_{j, 1}\right)+\cdots+a_{j, k_{j}} \tilde{\Psi}\left(v_{j, k_{j}}\right)\right\}=u_{j} .
$$

Set $U_{j}=\bigcap_{u \in \operatorname{Gen}(J)} U_{j, u}$ for $j=1, \ldots, r$. Then $U_{j} \subset G L_{n_{j}}(K)$ is a nonempty Zariski open subset. Choose $\varphi=\left(\varphi_{1}, \ldots, \varphi_{r}\right) \in G$ with each $\varphi_{j} \in U_{j}$. Then, as we saw in (3), for every $u=u_{1} \cdots u_{r} \in \operatorname{Gen}(J)$ with each $u_{j} \in \bigwedge\left\langle V_{j}\right\rangle$, there are elements $f_{1}, \ldots, f_{r}$ in $\bigwedge\langle V\rangle$ satisfying that

(a) each $f_{j}$ is a linear combination of monomials in $\left\{\tilde{\Psi}(v): v \leq_{\mathrm{P}} u_{j}, \operatorname{Deg}(v)=\right.$ $\left.\operatorname{Deg}\left(u_{j}\right)\right\} \subset \mathcal{N}\left\langle V_{j}\right\rangle$

(b) $\operatorname{in}_{\prec} \varphi_{j}\left(f_{j}\right)=u_{j}$.

Then $g=f_{1} f_{2} \cdots f_{r}$ satisfies $\operatorname{in}_{\prec} \varphi(g)=\left\{\operatorname{in}_{\prec} \varphi_{1}\left(f_{1}\right)\right\} \cdots\left\{\operatorname{in}_{\prec} \varphi_{r}\left(f_{r}\right)\right\}=u$ and $g$ is a linear combination of monomials in $\{\tilde{\Psi}(v): v \in \mathcal{G}\}$.

We will show that the set $\{\tilde{\Psi}(v): v \in \mathcal{G}\}$ is contained in $\tilde{\Psi}(J)$. Let $v \in \mathcal{G}$. Recall that $\tilde{\Psi}(v)=\pi\left(\operatorname{pol}\left(\tilde{\Phi}^{-1}\left(v^{\natural}\right)\right)\right)^{\mathrm{b}}$. It follows from Lemma $1.7(\mathrm{~g})$ that $\tilde{\Phi}^{-1}\left(v^{\natural}\right) \in$ $\tilde{\Phi}^{-1}\left(J^{\natural}\right)$. Then there exists a monomial $w \in \operatorname{Gen}(J)$ such that $\tilde{\Phi}^{-1}\left(w^{\natural}\right)$ divides $\tilde{\Phi}^{-1}\left(v^{\natural}\right)$. By the definition of the polarization, $\tilde{\Psi}(w)=\pi\left(\operatorname{pol}\left(\tilde{\Phi}^{-1}\left(w^{\natural}\right)\right)\right)^{b}$ divides $\tilde{\Psi}(v)$. Thus $\tilde{\Psi}(v) \in \tilde{\Psi}(J)$ for all $v \in \mathcal{G}$.

The above fact implies that $g \in \tilde{\Psi}(J)$ and $\operatorname{in}_{\prec} \varphi(g)=u \in \operatorname{in}_{\prec} \varphi(\tilde{\Psi}(J))$. Hence $u \in \operatorname{in}_{\prec} \varphi(\tilde{\Psi}(J))$ for all $u \in \operatorname{Gen}(J)$ as desired.

Remark 2.10 Let $I \subset K\left[V_{1}\right]$ be a strongly stable ideal. The generic initial ideal of $\operatorname{pol}(I)$ w.r.t. the reverse lexicographic order was determined in [6]. On the other hand, Proposition 2.9 determines the generic initial ideal of $\operatorname{pol}^{*}\left(\Phi(I)^{b}\right)=\operatorname{pol}(I)^{b}$ w.r.t. the reverse lexicographic order in the exterior algebra. In particular, this result determines the exterior algebraic shifted complex (see [13]) of some nontrivial simplicial complexes. For example, if $\Gamma$ is the simplicial complex defined by $I_{\Gamma}=\operatorname{pol}\left(\left\langle x_{1}, x_{2}, \ldots, x_{n}\right\rangle^{t}\right) \subset K\left[x_{i,[j]}: 1 \leq i \leq n, 1 \leq j \leq t\right]$, then it is known that $\Gamma$ is a simplicial ball (see [14, Theorem 3.1]). Then we can easily determine the exterior algebraic shifted complex of those simplicial balls.

One may expect a similar relationship for the generic initial ideal of $\Phi(I)$ and that of $\operatorname{pol}(I)$ when $I$ is not strongly stable. However, $\operatorname{pol}(I)$ and $\Phi(I)$ do not have such a nice relationship when $I$ is not strongly stable. Indeed, if $I=\left\langle x_{1}^{2}, x_{2}^{2}\right\rangle$ then $\operatorname{pol}(I)$ and $\Phi(I)=\left\langle x_{1} x_{2}, x_{2} x_{3}\right\rangle$ do not have the same Hilbert function.

\section{The proof of Theorem 0.1}

In this section, we will give a proof of Theorem 0.1 . Let, as before, $V=\dot{U}_{j=1}^{r} V_{j}$ be a set of variables with $V_{j}=\left\{x_{j, 1}, \ldots, x_{j, n_{j}}\right\}$ for $j=1, \ldots, r$. Throughout this section, we set $S=K[V]$ and $E=\bigwedge\langle V\rangle$. First, we recall two known results. See [13, Theorem 3.1] and [1, Proposition 2.1]. 
Lemma 3.1 Let $A=S$ or $A=E$ and $J$ a graded ideal of $A$. For any admissible order $\prec$, one has $\beta_{i j}^{A}\left(\right.$ in $\left._{\prec}(J)\right) \geq \beta_{i j}^{A}(J)$ for all $i$ and $j$.

Lemma 3.2 (Aramova-Avramov-Herzog) Let $J \subset E$ be a monomial ideal. Then

$$
\sum_{i} \sum_{j} \beta_{i j}^{E}(E / J) t^{i} s^{j}=\sum_{i} \sum_{j} \beta_{i j}^{S}\left(S / J^{\natural}\right) \frac{t^{i} s^{j}}{(1-t s)^{j}} .
$$

Lemma 3.2 implies the following useful fact.

Corollary 3.3 Let I and $J$ be monomial ideals in E.

(i) $\beta_{i j}^{S}\left(I^{\natural}\right)=\beta_{i j}^{S}\left(J^{\natural}\right)$ for all $i$ and $j$ if and only if $\beta_{i j}^{E}(I)=\beta_{i j}^{E}(J)$ for all $i$ and $j$.

(ii) If $\beta_{i j}^{S}\left(I^{\natural}\right) \leq \beta_{i j}^{S}\left(J^{\natural}\right)$ for all $i$ and $j$ then $\beta_{i j}^{E}(I) \leq \beta_{i j}^{E}(J)$ for all $i$ and $j$.

Now, we will prove Theorem 0.1 .

Proof of Theorem 0.1 We may assume that each $\left|V_{j}\right|=n_{j}$ is sufficiently large. Then the squarefree strongly color-stable ideal $\tilde{\Phi}(I)^{b} \subset E$ satisfies the assumption of Proposition 2.9. Set $J=\tilde{\Psi}\left(\tilde{\Phi}(I)^{b}\right)^{\natural}$. Notice that $J=\pi(\operatorname{pol}(I))$ by the definition of $\tilde{\Psi}$. Then Proposition 2.9 and Lemma 3.1 say that

$$
\beta_{i j}^{E}\left(\tilde{\Phi}(I)^{\mathrm{b}}\right) \geq \beta_{i j}^{E}\left(J^{\mathrm{b}}\right) \quad \text { for all } i \text { and } j
$$

On the other hand, by Theorem 1.10 and Lemma 3.1, we have

$$
\beta_{i j}^{S}(I) \geq \beta_{i j}^{S}(\tilde{\Phi}(I)) \quad \text { for all } i \text { and } j
$$

Since $I$ and $\operatorname{pol}(I)$ have the same graded Betti numbers, $I$ and $J=\pi(\operatorname{pol}(I))$ have the same graded Betti numbers. Hence (5) says

$$
\beta_{i j}^{S}(J) \geq \beta_{i j}^{S}(\tilde{\Phi}(I)) \quad \text { for all } i \text { and } j
$$

Then Corollary 3.3 (ii) says that $\beta_{i j}^{E}\left(J^{b}\right) \geq \beta_{i j}^{E}\left(\tilde{\Phi}(I)^{b}\right)$ for all $i$ and $j$. Thus, by (4), $J^{b} \subset E$ and $\tilde{\Phi}(I)^{b} \subset E$ have the same graded Betti numbers, and Corollary 3.3 (i) says that $J \subset S$ and $\tilde{\Phi}(I) \subset S$ have the same graded Betti numbers. Since $I$ and $J=\pi(\operatorname{pol}(I))$ have the same graded Betti numbers, the claim follows.

Corollary 3.4 Let $K$ be a field of characteristic $0, \Gamma$ a simplicial complex on $V$ and $\prec$ an admissible order. Then $\beta_{i j}^{K[V]}\left(I_{\tilde{\Delta}_{\prec}(\Gamma)}\right)=\beta_{i j}^{K[V]}\left(G-\operatorname{gin}_{\prec}\left(I_{\Gamma}\right)\right)$ for all $i$ and $j$.

Proof The statement immediately follows from Corollary 1.6 and Theorem 0.1 .

Example 3.5 Let $V_{1}=\left\{x_{1}, x_{2}, x_{3}, x_{4}\right\}, V_{2}=\left\{y_{1}, y_{2}, \ldots, y_{5}\right\}$ and $V=V_{1} \cup V_{2}$. Set

$$
I=\left(x_{1}^{3}, y_{1}^{4}, y_{1}^{3} y_{2}, y_{1}^{2} y_{2}^{2}, x_{1}^{2} y_{1}^{2}, x_{1} x_{2} y_{1}^{2}, x_{1}^{2} y_{1} y_{2}, x_{1} x_{2} y_{1} y_{2}\right)
$$


Then $I$ is strongly color-stable and

$$
\begin{aligned}
\tilde{\Phi}(I)= & \left(x_{1} x_{2} x_{3}, y_{1} y_{2} y_{3} y_{4}, y_{1} y_{2} y_{3} y_{5}, y_{1} y_{2} y_{4} y_{5}, x_{1} x_{2} y_{1} y_{2}, x_{1} x_{3} y_{1} y_{2},\right. \\
& \left.x_{1} x_{2} y_{1} y_{3}, x_{1} x_{3} y_{1} y_{3}\right) .
\end{aligned}
$$

By Theorem 0.1, the Betti diagram of $I$ and that of $\tilde{\Phi}(I)$ coincide. It is

\begin{tabular}{ccccc} 
& 0 & 1 & 2 & 3 \\
\hline $3:$ & 1 & - & - & - \\
4: & 7 & 8 & 2 & - \\
5: & - & 6 & 7 & 2 \\
total: & 8 & 14 & 9 & 2
\end{tabular}

(In the diagram the element at the $i$-th column and $j$-th row is $\beta_{i i+j}$.)

\section{Betti numbers and colored algebraic shifting}

In this section, we give an example of a color-shifted complex which shows some important facts on colored algebraic shifting.

Let, as before, $V=\dot{U}_{j=1}^{r} V_{j}$ with $V_{j}=\left\{x_{j, 1}, x_{j, 2}, \ldots, x_{j, n_{j}}\right\}$. A simplicial complex is called pure if all its faces have the same degree. Let $\Gamma$ be a simplicial complex and $\tilde{H}_{i}(\Gamma ; K)$ the reduced homology groups of $\Gamma$ with respect to the field $K$. The integers $b_{i}(\Gamma)=\operatorname{dim}_{K} \tilde{H}_{i}(\Gamma ; K)$ are called the Betti numbers of $\Gamma$. Since symmetric algebraic shifting (that is, colored algebraic shifting in the case of $r=1$ ) preserves Betti numbers, it was asked in [5] whether there exists an admissible order $\prec$ such that $b_{i}(\Gamma)=b_{i}\left(\tilde{\Delta}_{\prec}(\Gamma)\right)$ for all balanced complexes $\Gamma$. However, the next example shows that there are no such admissible orders. (Note that Hochster's formula [10, Theorem 5.5.1] and Corollary 3.4 imply $b_{i}(\Gamma) \leq b_{i}\left(\tilde{\Delta}_{\prec}(\Gamma)\right)$.)

Example 4.1 Let $V_{1}=\left\{x_{1}, x_{2}, x_{3}\right\}, V_{2}=\left\{y_{1}\right\}, V_{3}=\left\{z_{1}\right\}$ and $V=\bigcup_{j=1}^{3} V_{j}$. Set $\Gamma=$ $\left\langle x_{3} y_{1} z_{1}, x_{1} y_{1}, x_{2} z_{1}\right\rangle$. Then $\Gamma$ is a completely balanced complex on $V$ and $b_{i}(\Gamma)=0$ for all $i$ (see Fig. 1 below). Let $\Sigma=\left\langle x_{3} y_{1} z_{1}, x_{2} y_{1}, x_{2} z_{1}, x_{1}\right\rangle$. Then $\Sigma$ is a colorshifted complex with the same flag $f$-vector as $\Gamma$ and $b_{0}(\Sigma)=b_{1}(\Sigma)=1$.

However, it is easy to see that $\Sigma$ is the only color-shifted complex on $V$ with the same flag $f$-vector as $\Gamma$. Indeed, if $\Delta$ is a color-shifted complex on $V$ with the same flag $f$-vector as $\Gamma$ then $\Delta$ must contain $x_{3} y_{1} z_{1}$ since $f_{(1,1,1)}(\Gamma)=1$ and must contain $x_{2} y_{1}$ and $x_{2} z_{1}$ since $f_{(1,1,0)}(\Gamma)=f_{(1,0,1)}(\Gamma)=2$.

Fig. 1 A nonpure balanced complex and its colored algebraic shifted complex which do not have the same Betti numbers

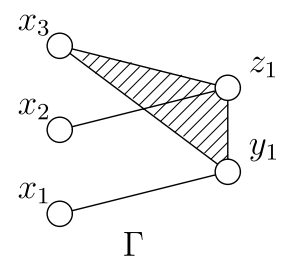




This fact says that $\tilde{\Delta}_{\prec}(\Gamma)=\Sigma$ for any admissible order $\prec$ but $b_{1}(\Gamma)<b_{1}(\Sigma)$. (More generally, we can replace $\tilde{\Delta}_{\prec}(-)$ by any operation $\Delta(-)$ satisfying $(\mathrm{C} 1)$ and (C2).)

The above example also implies another fact. It was stated in [5, Theorem 5.7] that if $\Delta$ is a balanced color-shifted complex on $V$ then

$$
b_{i-1}(\Delta)=\mid\left\{u \in \operatorname{Facet}(\Delta): \operatorname{deg}(u)=i \text { and } u \text { is not divisible by } x_{j, n_{j}} \forall j\right\} \mid
$$

where $\operatorname{Facet}(\Delta)$ is the set of facets of $\Delta$. Now, in the above example, $\Sigma$ is a completely balanced color-shifted simplicial complex and all its facets of degree 2 are divisible by $y_{1}$ or $z_{1}$. Then, since $V_{2}=\left\{y_{1}\right\}$ and $V_{3}=\left\{z_{1}\right\}$, the right-hand-side of (6) is 0 if $i=2$. However Fig. 1 says that $b_{1}(\Sigma)=1$. Hence (6) is a misstatement. The error appeared in the third line of the proof of [5, Theorem 5.7]. They stated that, for any $L \subset[r], \bigcap_{i \in L} \operatorname{st}_{\Gamma}\left(x_{i, n_{i}}\right)=\operatorname{st}_{\Gamma}\left(\prod_{i \in L} x_{i, n_{i}}\right)$, where $\operatorname{st}_{\Gamma}(v)=\left\{v^{\prime} u\right.$ : $v^{\prime}$ divides $v$ and $v u \in \Gamma$ \}. However, this is not true if $\Gamma$ is not pure. Indeed, in Fig. 1, $\operatorname{st}_{\Sigma}\left(y_{1}\right) \cap \operatorname{st}_{\Sigma}\left(z_{1}\right)=\left\langle x_{2}\right\rangle \cup\left\langle x_{3} y_{1} z_{1}\right\rangle$.

Actually equation (6) holds if $\Delta$ is a pure balanced color-shifted complex. Indeed it is not hard to see that the proof in [5] works under this assumption. We also notice that the above misstatement does not affect to other statements of [5] since (6) was used only for pure balanced color-shifted complexes. Babson and Novik [5] used the Nerve Theorem, which is a topological technique, for the proof of (6). In the rest of this section, we give a combinatorial proof of this equation for pure balanced colorshifted complexes.

A simplicial complex $\Gamma$ is called shellable if its facets can be ordered $F_{1}$, $F_{2}, \ldots, F_{k}$ so that $\left\langle F_{1}, \ldots, F_{i-1}\right\rangle \cap\left\langle F_{i}\right\rangle$ is generated by monomials of degree $\operatorname{deg}\left(F_{i}\right)-1$ for all $i>1$ (we do not assume that $\Gamma$ is pure). The order $F_{1}, F_{2}, \ldots, F_{k}$ is called a shelling of $\Gamma$.

Proposition 4.2 Let $\mathbf{a} \in \mathbb{Z}_{>0}^{r}$. If $\Gamma$ is a pure $\mathbf{a}$-balanced color-shifted complex on $V$ then $\Gamma$ is shellable and hence satisfies (6).

Proof Consider an order $F_{1}, F_{2}, \ldots, F_{k}$ of facets of $\Gamma$ satisfying $F_{i} \leq_{\mathrm{P}} F_{j}$ if $j \leq i$. It is clear that there exists such an order. We will show that this order is a shelling.

For each facet $F$ of $\Gamma$, let $d_{j}(F)$ be the largest integer $0 \leq t \leq n_{j}$ such that $x_{j, t}$ does not divide $F$ for $j=1,2, \ldots, r$, and set $D(F)=\left\{x_{s, t} \in V: t<\right.$ $d_{s}(F), x_{s, t}$ divides $\left.F\right\}$. Let $\Delta_{i}=\left\langle F_{1}, \ldots, F_{i}\right\rangle$ for $i \geq 1$. We claim that $\Delta_{i-1} \cap\left\langle F_{i}\right\rangle$ is generated by $W=\left\{F_{i} / x_{s, t}: x_{s, t} \in D\left(F_{i}\right)\right\}$ for all $1<i \leq k$.

First, we will show that $W \subset \Delta_{i-1} \cap\left\langle F_{i}\right\rangle$. Let $x_{j, t} \in D\left(F_{i}\right)$. Since $\Gamma$ is pure

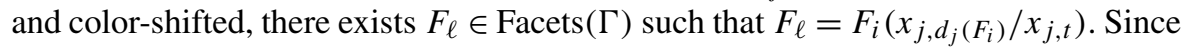
$F_{\ell}>_{\mathrm{P}} F_{i}$, the assumption on the order of facets implies that $\ell<i$. Hence $F_{\ell} \in \Delta_{i-1}$

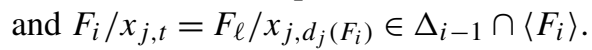

Next, we will show $\langle W\rangle \supset \Delta_{i-1} \cap\left\langle F_{i}\right\rangle$. Let $u \in \Delta_{i-1} \cap\left\langle F_{i}\right\rangle$. Suppose $u \notin\langle W\rangle$. Set $G_{1}=\prod_{j=1}^{r}\left(x_{j, d_{j}\left(F_{i}\right)+1} \cdots x_{j, n_{j}}\right)$ and $G_{2}=\prod_{v \in D\left(F_{i}\right)} v$. Hence $F_{i}=G_{1} G_{2}$. Then, since $u$ divides $F_{i}$ and $u \notin\langle W\rangle$, if follows that $G_{2}$ divides $u$. Since $u \in \Delta_{i-1}$, there exists $1 \leq t<i$ such that $u$ divides $F_{t}$. However, since $\operatorname{Deg}\left(F_{t}\right)=\operatorname{Deg}\left(F_{i}\right)$, 
we have $G_{1} \geq_{\mathrm{P}}\left(F_{t} / G_{2}\right)$ by the construction of $G_{1}$. Hence $F_{t} \leq_{\mathrm{P}} F_{i}$ but $t<i$. This contradicts the assumption on the order of facets.

Thus $\Gamma$ is shellable with the shelling $F_{1}, F_{2}, \ldots, F_{k}$. Also equation (6) immediately follows from this shelling (see e.g., [9, Theorem 4.1]).

Finally, we give a completely balanced color-shifted complex which is not shellable. Let $V_{1}=\left\{x_{1}, x_{2}\right\}, V_{2}=\left\{y_{1}\right\}, V_{3}=\left\{z_{1}\right\}, V_{4}=\left\{u_{1}\right\}, V_{5}=\left\{v_{1}\right\}$ and $V=$ $\bigcup_{j=1}^{5} V_{j}$. Set $\Gamma=\left\langle x_{1} y_{1} z_{1} u_{1} v_{1}, x_{2} y_{1} z_{1}, x_{2} u_{1} v_{1}\right\rangle$. This simplicial complex is completely balanced and color-shifted, however, is not shellable. Indeed, if $F_{1}, F_{2}, F_{3}$ is a shelling then we may assume that $F_{1}=x_{1} y_{1} z_{1} u_{1} v_{1}$ (e.g. by [9, Lemma 2.6]) and $F_{3}=x_{2} u_{1} v_{1}$ by the symmetry. However $\left\langle x_{1} y_{1} z_{1} u_{1} v_{1}, x_{2} y_{1} z_{1}\right\rangle \cap\left\langle x_{2} u_{1} v_{1}\right\rangle=$ $\left\langle x_{2}, u_{1} v_{1}\right\rangle$.

Acknowledgements I would like to thank Isabella Novik for useful discussions and for explaining the error in the proof of [5, Theorem 5.7]. I would also like to thank the referee for careful reading and helpful suggestions. The results and examples presented in this paper have been inspired by computations performed by the computer algebra system CoCoA [11].

\section{References}

1. Aramova, A., Avramov, L.L., Herzog, J.: Resolutions of monomial ideals and cohomology over exterior algebras. Trans. Am. Math. Soc. 352(2), 579-594 (2000)

2. Aramova, A., Crona, K., De Negri, E.: Bigeneric initial ideals, diagonal subalgebras and bigraded Hilbert functions. J. Pure Appl. Algebra 150(3), 215-235 (2000)

3. Aramova, A., Herzog, J., Hibi, T.: Gotzmann theorems for exterior algebras and combinatorics. J. Algebra 191, 174-211 (1997)

4. Aramova, A., Herzog, J., Hibi, T.: Shifting operations and graded Betti numbers. J. Algebra Comb. 12(3), 207-222 (2000)

5. Babson, E., Novik, I.: Face numbers and nongeneric initial ideals. Electron. J. Comb. 11(2), 23 (2006) Research Paper 25

6. Bigatti, A.M., Conca, A., Robbiano, L.: Generic initial ideals and distractions. Commun. Algebra 33(6), 1709-1732 (2005)

7. Björner, A., Frankl, P., Stanley, R.P.: The number of faces of balanced Cohen-Macaulay complexes and generalized Macaulay theorem. Combinatorica 7, 23-34 (1987)

8. Björner, A., Kalai, G.: An extended Euler-Poincaré theorem. Acta. Math. 161, 279-303 (1988)

9. Björner, A., Wachs, M.: Shellable nonpure complexes and posets. I. Trans. Am. Math. Soc. 348(4), 1299-1327 (1996)

10. Bruns, W., Herzog, J.: Cohen-Macaulay rings, Revised edn. Cambridge University Press, Cambridge (1998)

11. CoCoA Team: CoCoA: a system for doing computations in commutative algebra. Available at http://cocoa.dima.unige.it

12. Frankl, P., Füredi, Z., Kalai, G.: Shadows of colored complexes. Math. Scand. 63, 169-178 (1988)

13. Herzog, J.: Generic initial ideals and graded Betti numbers. In: Hibi, T. (ed.) Computational Commutative Algebra and Combinatorics. Advanced Studies in Pure Math, vol. 33, pp. 75-120 (2002)

14. Hibi, T., Singla, P.: Linear balls and the multiplicity conjecture. Preprint arXiv:0705.3531 (2007)

15. Kalai, G.: Algebraic shifting. In: Hibi, T. (ed.) Computational Commutative Algebra and Combinatorics. Advanced Studies in Pure Math, vol. 33, pp. 121-163 (2002)

16. Murai, S.: Generic initial ideals and squeezed spheres. Adv. Math. 214, 701-729 (2007)

17. Stanley, R.P.: Balanced Cohen-Macaulay complexes. Trans. Am. Math. Soc. 249(1), 139-157 (1979)

18. Stanley, R.P.: Combinatorics and Commutative Algebra, 2nd edn. Birkhäuser, Boston (1996) 